\title{
Cadáver exquisito poético: politizando los géneros literarios mediante estrategias didácticas
}

Poetic exquisite corpse: politicizing literary genders through didactic strategies.

\author{
Cadáver exquisito poético: Politizando os gêneros literários através \\ estratégias didáticas.
}

Keylor Robles Murillo

Universidad de Costa Rica

Alajuela, Costa Rica

robleskeylor@gmail.com

(D) https://orcid.org/0000-0002-1493-5582

Recibido - Received - Recebido: 20/03/2020ＣＣＣｒregido-Revised - Revisado:26/08/2020Ａceptado - Accepted - Aprovado: 13/10/2020

DOI: https://doi.org/10.22458/ie.v22i33.2845

URL: https://revistas.uned.ac.cr/index.php/innovaciones/article/view/2845

\begin{abstract}
Resumen: En el presente ensayo se expone el proceso de desarrollo de la estrategia didáctica titulada "Cadáver exquisito poético", la cual se llevó a cabo como parte de las evaluaciones sumativas y formativas del curso ED0012 Didáctica General de la Universidad de Costa Rica, Sede Occidente. El objetivo de este documento consiste en presentar una estrategia basada en la creatividad, en donde se articulan premisas de surrealismo con el fin de concretar una obra colectiva que permita la reflexión grupal. De igual manera, se señalan las implicaciones en dos ámbitos: a) a nivel pedagógico desde una perspectiva crítica y b) en el área específica de la Enseñanza del Español. Finalmente, se comparten las conclusiones obtenidas a partir del análisis.
\end{abstract}

Palabras clave: Didáctica, Pedagogía, Transformación Social, Literatura.

\begin{abstract}
The present essay puts forward the process and development of the didactic strategy entitled "Poetic exquisite corpse," which was carried out as part of the formative and summative evaluations of the course ED0012 Didáctica General at Universidad de Costa Rica, Sede Occidente. The purpose of this text is to present a creativity-based strategy where the postulates of surrealism are articulated in order to consolidate a collective work that allows for group reflection. In like manner, its implications in two different directions are discussed: a) the pedagogical level from a critical perspective and b) in the specific area of Spanish teaching. Finally, conclusions are shared.
\end{abstract}

Keywords: Didactics, pedagogy, social transformation, literature.

\section{(ब) (1) $\Theta$}


Resumo: Este ensaio apresenta o processo de desenvolvimento da estratégia didática intitulada "Cadáver exquisito poético", que foi realizado como parte das avaliações somativas e formativas do curso ED0012 Didática Geral da Universidad de Costa Rica, Sede Ocidente. O objetivo deste documento é apresentar uma estratégia baseada na criatividade, onde são articuladas premissas de surrealistas a fim de criar um trabalho coletivo que permita a reflexão coletiva. Da mesma forma, as implicações em duas áreas: a) no nível pedagógico a partir de uma perspectiva crítica e b) na área específica do Ensino da Língua Espanhola. Finalmente, as conclusões obtidas a partir da análise são compartilhadas.

Palavras-chave: Didática, pedagogia, transformação social, literatura. 


\section{INTRODUCCIÓN}

La educación al estar vinculada y, a su vez, circunscrita a un contexto cultural, social, político y económico determinado, ocasiona que las relaciones educativas establecidas entre todos los actores dentro de los ambientes áulicos, y en contextos no formales, se deban interpelar, resignificar y replantear de manera constante. Lo anterior, se tiene que desarrollar principalmente con el fin de construir, propiciar y facilitar estrategias didácticas novedosas que adecúen la forma en cómo se construye el conocimiento dentro de la coyuntura actual.

A partir del planteamiento anterior, se considera fundamental promover la consolidación de estrategias didácticas que tomen en cuenta las realidades y las particularidades en que se ubica el estudiantado. Lo anterior, debido a que la finalidad de la didáctica corresponde a diseñar estrategias que promuevan en las personas la búsqueda y construcción de experiencias de aprendizajes significativos desde perspectivas críticas (Picado, 2006), en donde los actores implicados en el acto educativo logren comprenderse como sujetos históricos y socioculturales.

En el presente ensayo, se exponen los fundamentos, la conceptualización y el proceso metodológico de la estrategia didáctica denominada "Cadáver exquisito poético", la cual se desarrolló colectivamente durante el año 2018 como parte de las evaluaciones sumativas y formativas del curso ED0012 Didáctica General; enmarcado en las mallas curriculares de las carreras del Departamento de Educación de la Universidad de Costa Rica, Sede de Occidente.

\section{Aspectos preliminares}

Inicialmente, se torna pertinente señalar que en el documento se emplea el término estrategia didáctica para referirse al "Cadáver exquisito poético", ya que se parte de la conceptualización propuesta por Zúñiga (2017), quien lo define de la siguiente manera:

[...] un procedimiento organizado, formalizado y orientado para la obtención de una meta claramente establecida. Su aplicación en la práctica requiere del perfeccionamiento de procedimientos y de técnicas cuya elección detallada y diseño son responsabilidad del cuerpo docente, quien elige las técnicas y actividades y las decisiones que debe tomar de manera consciente y reflexiva. (p. 3)

Con respecto a este aporte teórico, se destacan tres aspectos relacionados directamente con la propuesta del "Cadáver exquisito poético". En primer lugar, la estrategia didáctica planteada se desarrolla mediante momentos metodológicos concatenados, que se explican más adelante. En segundo lugar, en esta oportunidad se establece un objetivo pedagógico que guía el proceso de enseñanza y aprendizaje. En tercer lugar, su perfeccionamiento se encuentra vinculado con la aplicación en diversos contextos. No obstante, es importante aclarar que el autor del texto no cree que en dichas estrategias la responsabilidad recae exclusivamente en el cuerpo docente.

Del mismo modo, se considera que la estrategia didáctica expuesta es congruente con la pedagogía contestataria, esta última entendida como "antítesis y ejercicio de resistencia ante la influencia del capitalismo en la educación, y a su vez, como alternativa a las relaciones de poder adultocéntricas; pues su objetivo consiste en desarrollar una transformación integral dentro del sistema educativo tradicional" (Robles, 2019, p. 52). Además, también se articula con sus fundamentos, los cuales se resumen en la figura 1: 
Figura 1. Fundamentos de la pedagogía contestataria

\begin{tabular}{|c|c|}
\hline Epistemológico & $\begin{array}{l}\text { - La construcción del conocimiento es un acto político. } \\
\text { - Parte de tres enfoques: intelectual, feminista y Queer. }\end{array}$ \\
\hline Ontológico & $\begin{array}{l}\text { - La realidad se analiza desde la dialéctica. } \\
\text { - Se resalta el papel protagonista del estudiantado. }\end{array}$ \\
\hline Axiológico & $\begin{array}{l}\text { - Se toman como fundamento los siguientes valores: } \\
\text { igualdad, equidad, respeto, compromiso, solidaridad, } \\
\text { diversidad, sororidad, redistribución y justicia social. }\end{array}$ \\
\hline Teleológico & $\begin{array}{l}\text { - El fin primordial de la educación debe relacionarse con } \\
\text { fortalecer la liberación del pueblo oprimido. }\end{array}$ \\
\hline Metodológico & $\begin{array}{l}\text { - Estrategias didácticas innovadoras, que fomenten el } \\
\text { pensamiento crítico y la curiosidad. }\end{array}$ \\
\hline
\end{tabular}

Fuente: Elaboración propia a partir de Robles (2019).

Vale mencionar que en este ensayo se profundiza en los fundamentos metodológicos, ya que algunas pedagogías se reducen a discusiones teóricas sin brindar aportes sobre el proceso de ejecución. Sin embargo, no se dejan de lado los otros fundamentos de la pedagogía contestataria ni sus antecedentes: los aportes de la pedagogía del oprimido (Freire, 1982), la pedagogía de la autonomía (Freire, 2006) y la pedagogía crítica (Giroux, 2003; McLaren, 2012). A continuación, se exponen cuáles son los supuestos incorporados y cómo se vinculan con la presente estrategia.

En primer lugar, la pedagogía del oprimido, creó un movimiento de base que le otorgó un carácter político al problema educativo. Además, convirtió la alfabetización en un mecanismo para concientizar al pueblo y aproximarlo críticamente a su realidad, mediante una visión dialógica o conversacional. Desde estas premisas, también los educandos se vuelven protagonistas en el proceso de enseñanza-aprendizaje, ya que se conciben como sujetos capaces de originar una nueva dinámica educativa orientada hacia la problematización (Freire, 1982; Ocampo, 2008). Lo anterior, cobra relevancia dentro de esta estrategia didáctica, pues reitera la importancia de politizar otros espacios o ámbitos que han sido mostrados, por los grupos de poder, como si fuesen neutrales.

En segundo lugar, respecto a la pedagogía de la autonomía, esta parte del diálogo igualitario y solidario, en donde se rompe con posturas jerárquicas y autoritarias de la educación tradicional o bancaria, ya que todas las personas que intervienen en el proceso educativo, docentes y estudiantes, generan procesos de comunicación horizontales. Sumado a esto, la pedagogía citada aboga por la transformación de la realidad, tomando en cuenta las vivencia situadas de cada persona (Freire, 2006; Flecha, 2004). En el "Cadáver exquisito poético", los participantes se involucran y relacionan desde la horizontalidad, es decir, ninguna contribución es más valiosa que otra.

En tercer lugar, en lo que concierne a la pedagogía crítica, se debe contemplar que esta se origina en congruencia con las contribuciones pedagógicas de Freire. Por consiguiente, mantiene una apuesta ética y política sustentada desde el reconocimiento, empoderamiento y democracia de los sujetos. Asimismo, esta pedagogía se constituye en un espacio para la denuncia y el anuncio: a) denuncia de las condiciones estructurales de desigualdad en todos sus órdenes y b) anuncio desde la posibilidad de implicarse con la 
otra persona (Giroux, 2003; McLaren, 2012; Ortega, 2009). Mediante la elaboración del poema, se planteó un tema central del cual se pudiesen desprender elementos sobre el derecho a la educación como forma de empoderamiento y ciudadanía activa.

Es oportuno indicar que si bien la presente estrategia consiste en un ejercicio didáctico que vincula contenidos curriculares abordados en la carrera de la Enseñanza del Castellano y la Literatura; puede ser replicado en las otras áreas de enseñanza (Estudios Sociales y Cívica, Educación Primaria, Educación Inicial, Matemáticas, Ciencias Naturales, entre otras) según sus objetivos de aprendizaje. Por esta razón, el objetivo de este ensayo es proponer una guía orientadora que es inacabada y se encuentra abierta a cambios según el contexto en que se desarrolle.

\section{Definición del "Cadáver exquisito"}

Antes de seguir, se debe mencionar que en la comunidad académica y artística no existe consenso sobre el motivo por el cuál dicha técnica, concebida como estrategia didáctica en esta oportunidad, lleva ese nombre. Empero, existen diversas conceptualizaciones que permiten un primer acercamiento teórico a su definición. En primer lugar, Breton (2003) señala lo siguiente:

[...] es una composición cooperativa entre varios individuos, para la creación de una obra a partir de sus colaboraciones, que contenga elementos o trozos de cada uno de ellos y que permita crear una obra única a partir de diferentes intencionalidades. (p.9)

Arqueros, Pérez y Richard (1990) añaden que consiste en una recopilación "de fragmentos que, alejados de sus relatos, se interconectan en posibles abiertos [...] siendo una acumulación de señas de nuestras historias nunca terminadas" (p.3). Para efectos de este ensayo, el "Cadáver exquisito" se entiende como una creación colectiva y horizontal basada en la espontaneidad, la creatividad y la socialización de aportes, cuyo fin principal consiste en la generación de un producto integrador de las múltiples perspectivas y las visiones de mundo.

En esta misma línea, Saba (2019) alude a que en el "Cadáver exquisito" los aspectos completamente accidentales juegan un papel crucial. Además, en las creaciones desarrolladas de este modo, los nombres de los autores son suprimidos, ya que la obra se concibe como una creación colectiva en la que predomina la combinación contingente de las partes que la conforman. Algunos autores en la literatura latinoamericana que han empleado esta técnica son los siguientes: a) en Argentina, Jorge Luis Borges (1899-1986) y Adolfo Bioy Casares (1914-1999), bajo el seudónimo común de H. Bustos Domecq, escribieron Seis problemas para Don Isidro Parodi (1942), b) en Perú, César Vallejo (1892-1938), poeta peruano, escribió Poemas humanos (1938, en Hart, 2018).

En lo que refiere al origen del "Cadáver exquisito", Álamo (2017) indica que, al igual que sucede con la imprecisión de las razones que explican su nombre, no existe completa claridad sobre su origen. Sin embargo, el autor menciona que surgió a partir de los planteamientos de los primeros artistas surrealistas en la década de 1920: a) literatura: André Breton (1896-1966), Louis Aragón (1897-1982) y Paul Éluard (1895-1952); y b) iconografía: Luis Buñuel (1900-1983), Salvador Dalí (1904-1989) y Max Ernst (1891-1976). En esta corriente también se ubican Frida Kahlo (1907-1954), René Magritte (1898-1967), Dorothea Tanning (1910-201) y Pablo Picasso (1881-1973).

El surrealismo al ser una corriente y un movimiento artístico abstracto que se extendió en literatura y la iconografía, provocó que las técnicas empleadas incluyeran elementos textuales y visuales. Por esta razón, actualmente los "cadáveres exquisitos" más empleados en las artes plásticas son los dibujos 
y el collage; mientras que en la literatura corresponden a los textos narrativos, mayoritariamente, cuentos por la particularidad de ser breves. En este caso, se decidió incursionar en la poesía, ya que la innovación debe ser un elemento transversal dentro de la práctica docente.

Para ampliar el análisis, se deben contemplar dos premisas que marcan las particularidades del "Cadáver exquisito poético". En primer lugar, si bien esta técnica se enmarca dentro de una tradición artística basada en categorías del psicoanálisis freudiano: inconsciente, psique, sueños, entre otras (Breton, 1924); en esta experiencia, se decidió concretar los grados de abstracción del surrealismo al enfocarlo en una situación de la vida cotidiana en el ambiente áulico. En segundo lugar, surgió como una práctica de creación artística y no como una estrategia didáctica. Han sido docentes de todos los niveles quienes se han comprometido con la innovación educativa y adecuaron la ejecución de la técnica para convertirla en un recurso de mediación pedagógica basado en la lúdica y la espontaneidad dentro del proceso educativo.

Respecto a la metodología para desarrollar esta técnica, Breton (2003) describe el proceso: "varias personas componen una frase o un dibujo en cada uno de los pliegues de un papel sin que ninguna de ellas pueda saber qué han escrito o dibujado las anteriores" (p.25). A lo anterior, se debe añadir que existen dos formas principales de llevarlo a cabo. En la primera, los aportes de cada persona se hacen tomando únicamente el elemento que precede. Por ejemplo, en los "cadáveres exquisitos" gráficos la guía es el último trazo realizado; mientras que en los textuales se orienta a partir de la última frase colocada. En la segunda manera, cada participante brinda su aporte sin observar nada de lo que han hecho las otras personas; solamente guiado por un tópico central.

A continuación, se muestra un ejemplo escrito en verso:

Poema 1. R-EVOLUCIÓN

$\begin{array}{ll}\text { Al principio } & \text { Hasta que la vida abrió } \\ \text { Fueron palabras brillantes: } & \text { Caminos } \\ \text { Azul, nostalgia } & \text { Reventó } \\ \text { Vocablos elevados: } & \text { Arrastró } \\ \text { Olivar, alcazaba } & \text { Normas tradiciones } \\ \text { Con-tacto suave } & \text { Ahora digo versos } \\ \text { Frialdad más tarde } & \text { Y habla la poesía } \\ \text { El dique agrieta } & \text { Con ecos de calles de periódico } \\ \text { p-a-l-a-b-r-a-s } & \text { Voz en zapatilla } \\ \text { MANCHADAS de realidad } & \text { Grito en vida plena }\end{array}$

Radios coches calvicies

Fuente: Sánchez y Valladolid (2008, p. 52).

Este poema colectivo es parte del capítulo titulado "A dos voces", del libro Antología El Cadáver exquisito: la creación colectiva como fin. En esta oportunidad, se mantiene la puntuación y el énfasis del texto original; no obstante, las palabras escritas en vertical, aquí se redactan horizontalmente. En este punto se deben contemplar dos aspectos: a) los autores no explican el contexto en que se desarrolló el proceso y b) al igual que la estrategia didáctica expuesta, el tema central del poema se relaciona con la transformación de la realidad, al pasar por tres etapas, que se detallan en la figura 2 : 
Figura 2. Etapas del poema R-EVOLUCIÓN

1. ${ }^{\circ}$ Etapa:
INICIO
Existe nostalgia,
frialdad; junto a una
realidad aplastante
que silencia.
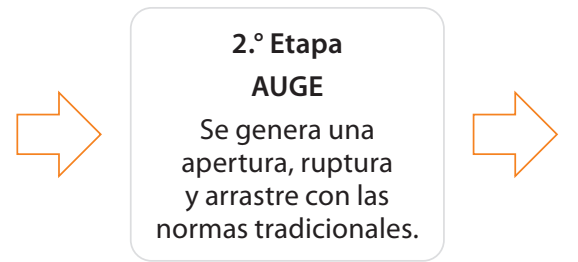

$3 .^{\circ}$ Etapa
CULMINACIÓN
Se recupera la voz
poética, resonante en
toda la ciudad. La vida
vuelve a ser plena.

Fuente: Elaboración propia.

Como se mencionó anteriormente, el "Cadáver exquisito", desde su origen, ha sido un proceso de creación vinculado con una tradición iconográfica. Por tal motivo, en este ensayo se considera pertinente incorporar el siguiente ejemplo gráfico (figura 3):

Figura 3. Construyendo (2006). Técnica: Mixta. Tamaño: 1,30 m x 1,89 m.

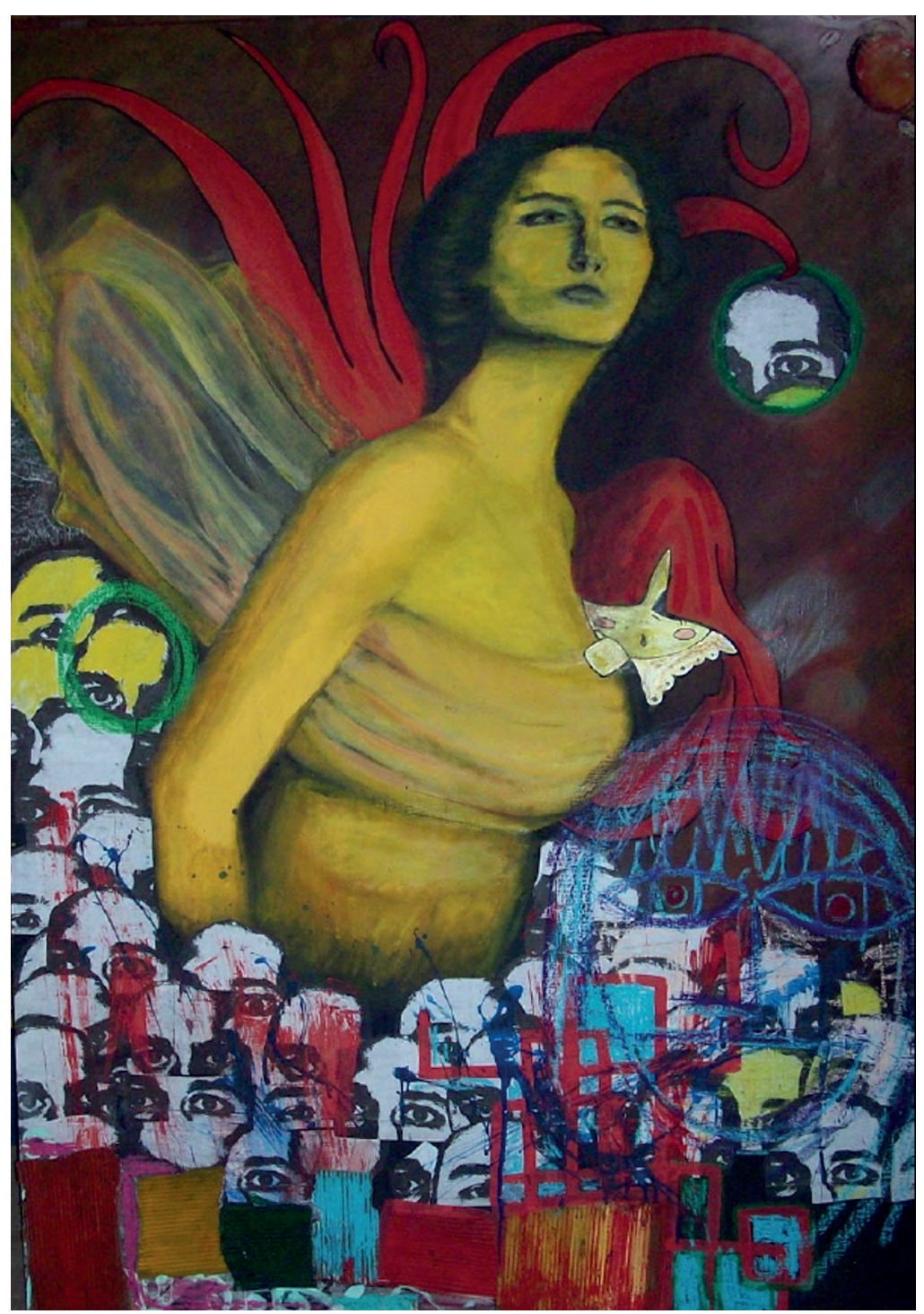

Fuente: Maza, Tourn, Casas, Maza, Ferreyra y López (2008, p. 37). 
Esta obra forma parte del mismo libro que el poema anterior. Es la única representación visual de la antología. A partir de la observación y análisis de dicha obra, se podría profundizar en el cuerpo de las mujeres como paradigma teórico y ontológico en el arte, y a su vez, como espacio de encuentros y desencuentros. Para ampliar estas ideas, se pueden revisar los aportes de Oliva (2017) y González (2018). Este par de ideas son solo esbozos de algunos aspectos que se podrían estudiar a partir de esta creación colectiva del "Cadáver exquisito".

\section{DESARROLLO DEL “CADÁVER EXQUISITO”}

Como se mencionó al inicio del ensayo, la estrategia didáctica descrita se llevó a cabo durante el segundo ciclo del año 2018 en el marco del curso ED0012 Didáctica general, el cual se caracteriza por lo siguiente:

[...] es teórico-práctico y aborda el conocimiento de la didáctica como disciplina esencial para los docentes. Establece además una relación entre esta, la pedagogía, la educación y el currículo. (Departamento de Ciencias de la Educación, 2018, p.1)

En el "Cadáver exquisito poético" se contó con la participación voluntaria de catorce estudiantes del curso, tanto hombres como mujeres, y de la docente del curso. El objetivo general que orientó esta estrategia fue el siguiente: "Reconocer la importancia de la creatividad y la espontaneidad en la elaboración de trabajos colectivos que se realizan en los escenarios educativos". Junto a este, se definieron los siguientes objetivos específicos:

1. Presentar la estrategia como un espacio que favorece la expresión diversa de los participantes y de los posteriores espectadores.

2. Identificar las formas en cómo las estrategias didácticas basadas en recursos literarios sirven para comprender críticamente la realidad y el contexto.

3. Demostrar los aportes pedagógicos que brinda la estrategia didáctica en el desarrollo cognitivo de los estudiantes participantes, 4. Elaborar de manera conjunta la estrategia con el fin de que pueda ser replicada en espacios cotidianos. (Robles, 2018)

Es oportuno aludir a que toda estrategia didáctica debe encontrarse fundamentada teórica y curricularmente, lo que implica una búsqueda en fuentes especializadas en materia de educación. Para este fin, se sustentó en los siguientes aportes: a) la concepción de la lúdica materializada en actividades de aprendizaje transformadoras (Jiménez, 1997); b) la importancia de la cooperación genuina en los escenarios educativos (Val de Velde, 2014); y c) el papel que desempeñan las vivencias del estudiantado en los procesos de generación de conocimiento (Aldana, 2011).

Seguidamente, se explicó el proceso para desarrollar la estrategia didáctica del "Cadáver exquisito", la cual se conforma por una serie de pasos. Para efectos de graficar la información principal, se decide agrupar el procedimiento en cuatro grandes momentos metodológicos que se exponen en la figura 4: 
Figura 4. Momentos metodológicos del "Cadáver exquisito poético"

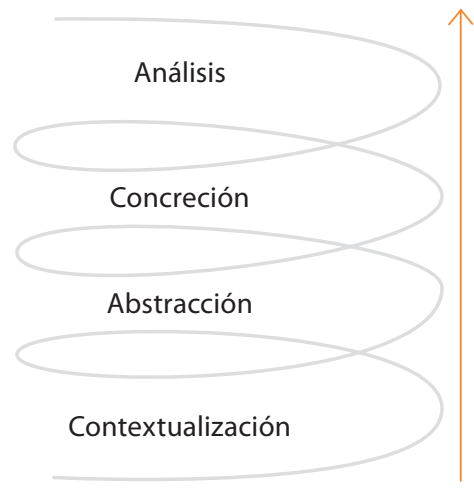

Fuente: Elaboración propia.

En primer lugar, la contextualización implica los siguientes pasos: a) explicación del procedimiento de la estrategia: el facilitador compartió al grupo la forma llevar a cabo la estrategia didáctica, b) la socialización de los objetivos: en este punto se profundizó en los objetivos definidos, debido a que la finalidad era proponer una estrategia que el estudiantado del curso pudiese replicar en un futuro ejercicio profesional como docentes de todos los niveles y áreas de aprendizaje, y c) la definición de los materiales requeridos: para la elaboración de la estrategia se requirió un pliego de papel periódico y un marcador de cualquier color.

Sumado a lo anterior, se explicitó que el tema central era "el derecho a la educación". En el siguiente apartado se profundiza los motivos por los cuales se seleccionó dicho tópico. Una de las recomendaciones brindadas al grupo fue que los versos no fueran mayores a ochos palabras; no obstante, esto no fue un impedimento para limitar la creatividad, sino más bien, la intención era garantizar la participación de todo el grupo sin monopolizar el uso del marcador.

En segundo lugar, la abstracción refiere al proceso en donde surge la creación colectiva, pues cada aporte se realiza desde la espontaneidad y la cooperación, sin conocer el resultado de manera global. Específicamente, este momento inició cuando la primera persona participante escribió una frase, en verso, que tomó como elemento orientador el título definido. En este caso la frase fue: "la maravilla de aprender". Es pertinente señalar que el fragmento puede incidir en el proceso de creación, pues en ocasiones, determina el rumbo de los demás aportes.

Seguidamente, la próxima persona, es decir, la segunda redactó un verso tomando como referencia el aporte brindado previamente. Después de finalizar el turno respectivo, cada estudiante dobló el pliego de papel con el objetivo de dejar visible únicamente el último verso, es decir, el que escribió cada quien. Esta dinámica se repitió hasta que todas las personas que deseaban participar, pudieron tener su turno para aportar a la obra colectiva.

En tercer lugar, el momento denominado concreción tiene el fin de trascender la abstracción surrealista para materializar una obra concreta. En este caso, comenzó cuando el facilitador desdobló el pliego de papel periódico para leer el poema de manera conjunta. Una sugerencia que se puede implementar en este proceso es solicitarle a una persona voluntaria que lo recite. "El Cadáver exquisito" construido en clase dio como resultado la siguiente creación: 
Poema 2. El derecho a la educación

1 La maravilla de aprender,

2 el tesoro más valioso que tienen las personas.

3 Un derecho que hace crecer nuestro corazón

4 motivando la creatividad,

5 vino a cambiar todo mi concepto

6 provocando un impacto en mí.

7 Transformando la vida y el mundo

8 con el conocimiento desde lo profundo
9 y una poesía que se mantiene en el aire,

10 y penetra al corazón de cada ser

11 rompiendo las barreras que posea

12 la ignorancia de muchos y la inocencia

13 de los dormidos.

14 Debemos despertar.

15 Romper las cadenas y volar.

Fuente: Obra colectiva (Robles, 2018).

En cuarto lugar, se desarrolló el momento analítico. En esta fase se pueden vincular las experiencias del estudiantado con elementos teóricos, epistemológicos, ontológicos y axiológicos. Por tanto, la estrategia debe contemplar un período de tiempo significativo para desarrollar el momento, con el propósito de generar reflexiones y discusiones colectivas. En el caso descrito, dicho análisis se llevó a cabo después de leer el poema. Además, se le solicitó al estudiantado que externaran sus impresiones sobre la estrategia y la obra elaborada conjuntamente.

Para efectos de ejemplificar cómo se puede analizar la poesía construida desde los fundamentos del análisis literario, se integran los planteamientos propuestos por Ramírez y Solano (2018), específicamente en la fase analítica. Si bien es cierto, resulta interesante aplicar toda la propuesta teórica-metodológica de estos autores al poema; en esta ocasión no se desarrolla para no desviarse del tópico central. Eventualmente, en futuros documentos se podría realizar.

En esta fase analítica interesa revisar la estructura, la retórica y la estilística, junto con el contexto. Cabe mencionar que el poema se enumeró por el autor de este ensayo con fines metodológicos para facilitar el análisis; no obstante, debe ser entendido como un texto unificado, el cual es mucho más que la suma de sus partes individuales. Además, se respetó la puntuación empleada por el grupo de personas participantes durante su creación.

Inicialmente, la estructura del poema se conforma por tres grandes momentos: a) la educación desde el ámbito cognitivo y formativo, b) la educación como motor de transformación personal y c) la educación como medio de transformación social y liberación. En primer lugar, la educación se analiza desde un escenario que brinda la oportunidad de generar conocimientos y saberes (vv 1-5). Se compara dicho derecho con un regalo invaluable al dar como resultado procesos creativos que permiten deconstruir el nivel conceptual adquirido previamente.

En segundo lugar, se percibe como un derecho que permite lograr transformaciones en el plano personal y social (vv 6-10). A partir de este punto, se dimensiona el origen de una praxis educativa, pues el conocimiento reconstruido se convierte en un insumo medular para encauzar transformaciones. Igualmente, se enfatiza en cómo la poesía puede volver perdurables los aprendizajes a lo largo de las décadas y, al mismo tiempo, socializarlos en las personas.

En tercer lugar, después del proceso reflexivo sobre la importancia de la educación, se pasa a un momento práctico, es decir, se proponen acciones concretas para defender este derecho (vv 11-15). El mensaje central del poema se consolida alrededor de la idea de la educación como un espacio colectivo para 
superar la ignorancia, voluntaria o inocente, que mantiene a la población dormida ante su contexto. Incluso el poema finaliza haciendo un llamado a las lectoras y los lectores: "Debemos despertar / Romper las cadenas y volar". Similar a la estrategia empleada por Oreamuno (1938), quien al final de su ensayo "¿Qué hora es?", expresa que: "[...] debemos aunar esfuerzos tras un objetivo que borre diferencias de posiciones económicas, vitalizando la enseñanza, universalizándola, para así lograr que tenga verdadero contenido social [...]" (p. 9).

Respecto a la retórica y estilística del poema se puede enfatizar en el uso de la metáfora y la analogía. La metáfora se representa cuando se manifiesta que la educación es el tesoro más valioso que tienen las personas (v.2), que debe ser cuidado y defendido. Mientras que la analogía se presenta en dos momentos: verso tres y verso diez. Ambas relaciones se establecen con el corazón, pues el fin es reflejar la capacidad de la educación para vincular los sentires y los saberes, al convertirse en un elemento central; al igual que el corazón con el cuerpo.

Finalmente, el poema se contextualiza dentro de una sociedad en donde las personas no analizan ni comprenden su realidad, debido a que se encuentran oprimidas. Por tanto, en este escenario la educación se convierte en el medio que permite desmontar el conocimiento autorizado y el sistema de valores dominantes. Al mismo tiempo, se transforma en el escenario idóneo para cambiar las relaciones asimétricas de poder. La capacidad transformadora lleva a enfatizar en la defensa de este derecho y, a su vez, se acompaña de acciones para liberarse, por lo que se promueve una praxis transformadora que abogue por una sociedad basada en la solidaridad.

\section{Implicaciones pedagógicas y críticas del "Cadáver exquisito poético"}

La estrategia didáctica abordada pretende generar un proceso de enseñanza y aprendizaje de forma dinámica y creativa, cuya finalidad reside en que el estudiantado potencialice sus propias ideas, a partir de los objetivos curriculares desarrollados en la clase. Para este propósito, es necesario reorientar la praxis educativa hacia un continuo proceso de construcción, deconstrucción y reconstrucción; en donde la autocrítica y la reflexión colectiva sean elementos centrales dentro del accionar de las personas que participan en el proceso. En esta ocasión, se considera que las fases metodológicas planteadas permiten fomentar estos objetivos.

Relacionado con lo anterior, el "Cadáver exquisito poético", al circunscribirse en los fundamentos de la pedagogía contestataria, intenta consolidarse en una estrategia didáctica que otorgue herramientas para luchar por la transformación de la realidad social, desde un micro escenario como lo es un aula. En el ensayo, se considera que lo anterior se alcanza mediante la promoción de un pensamiento crítico, en donde cada estudiante pueda forjar su propia visión del mundo y del contexto en que se ubica; rompiendo con la práctica homogeneizadora de la educación tradicional, la cual ha impuesto históricamente los modos y los esquemas de pensamiento oficiales.

En esta misma línea, desde esta estrategia didáctica también se trabajan dos valores fundamentales en la construcción de una sociedad basada en la justicia social: el respeto y la solidaridad. Como se mencionó en apartados anteriores, en el "Cadáver exquisito" ninguna contribución es más importante que otra, al contrario, los aportes cobran relevancia cuando se interconectan con otros en una obra final. Por tanto, este tipo de técnicas incentivan una perspectiva de la educación como un espacio de solidaridad y no de competencia según la lógica neoliberal. Además, permite la comprensión del estudiantado como sujetos sociales.

Otra de las implicaciones pedagógicas del "Cadáver exquisito poético" consiste en la vinculación de diferentes áreas de la cultura. En este caso, se articula una práctica artística, de una gran tradición iconográfica, 
con la literatura. La innovación se relaciona con lo expuesto por Picado (2006), quien hace alusión a que el profesorado debe implementar acciones pedagógicas congruentes con las capacidades y conocimientos previos del estudiantado, mediante los aportes y planteamientos de una didáctica resignificadora. Por tanto, el o la docente debe innovar en técnicas que abarquen los múltiples estilos de aprendizaje y potencialicen la creación de experiencias de aprendizajes significativos críticos. Según Moreira (2005) este tipo de experiencias:

[...] permite al sujeto formar parte de su cultura y, al mismo tiempo, estar fuera de ella [...] A través del aprendizaje significativo crítico es como el alumno podrá formar parte de su cultura y, al mismo tiempo, no ser subyugado por ella, por sus ritos, sus mitos y sus ideologías. Es a través de ese aprendizaje como el estudiante podrá lidiar, de forma constructiva, con el cambio, sin dejarse dominar. (pp. 87-88)

Generar procesos de enseñanza que contemplen los diferentes estilos de aprendizaje, permite garantizar que el estudiantado se vincule con el proceso educativo y los contenidos curriculares abordados; pues desarrollan un proceso de pertenencia e identificación. Del mismo modo, se debe añadir que la vinculación también logra que las personas se sientan protagonistas de sus procesos educativos, que sean capaces de cuestionar y desafiar la dominación, así como las prácticas, las creencias y los valores establecidos; acompañado de proponer alternativas de sociedades solidarias, justas e inclusivas. Para alcanzar este fin, también se debe retomar el papel activo del sujeto en el proceso de conceptualización y el reconocimiento de sus experiencias; el cual es central durante el proceso de elaboración del "Cadáver exquisito poético".

\section{Implicaciones didácticas del "Cadáver exquisito poético" en la Enseñanza del Español}

Como se adelantó, el tema planteado para la construcción del poema se definió como el derecho a la educación, debido a que la finalidad era convertir los géneros literarios y su elaboración en una oportunidad para abordar temas políticos y sociales. Cabe agregar que la estrategia se relaciona con los enfoques curriculares integrados en los Programas de Estudio de Español para Tercer Ciclo y Educación Diversificada, del Ministerio de Educación Pública (2017), de acuerdo con la tabla 1:

TABLA 1

Enfoques pedagógicos y curriculares de los Programas de Estudio de Español para Tercer Ciclo y Educación Diversificada (2017)

\begin{tabular}{l|l} 
Enfoque & Planteamientos \\
\hline Pedagogía crítica & $\begin{array}{l}\text { La educación debe convertirse en una instancia mediadora a través del diálogo respetuoso, } \\
\text { en donde exista libertad para que las personas aprendientes cuestionen las creencias y } \\
\text { las prácticas. }\end{array}$ \\
\hline Socioconstructivismo & $\begin{array}{l}\text { El aprendizaje como una actividad social en la colaboración y el intercambio con las demás } \\
\text { personas. El individuo no se encuentra solo, sino que se desarrolla dentro de una cultura } \\
\text { determinada. }\end{array}$ \\
\hline Teoría holista & $\begin{array}{l}\text { Una educación basada en el desarrollo integral y global de la persona que aprende como } \\
\text { un ser individual y que, a su vez, se percibe como un ciudadano o ciudadana global. }\end{array}$ \\
\hline Habilidades para la vida & $\begin{array}{l}\text { Permiten la toma de decisiones para plantear y definir las habilidades para la nueva ciuda- } \\
\text { danía y la propuesta de un perfil del estudiante y de la persona docente. }\end{array}$ \\
\hline
\end{tabular}

Fuente: Ministerio de Educación Pública (2017). 
Dentro de los programas educativos, se establece el siguiente eje temático: "El ser humano se comunica de diversas formas y en contextos distintos como medio de convivencia en la sociedad nacional y global, aprovechando todo tipo de recursos" (Ministerio de Educación Pública, 2017, p. 112). Junto a esto, se definen competencias específicas en tres áreas: a) oral, b) escrita y c) oral y escrita; las cuales se operacionalizan a través de las siguientes estrategias didácticas: redacciones, interpretaciones, lectura, análisis de textos, técnicas de comunicación oral (debates, foros, conversatorios, círculos de discusión, plenarias) y análisis de imagen. Por tanto, a partir de la discusión expuesta en el ensayo, se espera que se incluyan las creaciones literarias colectivas, como el "Cadáver exquisito" en poesía o narrativa, dentro de las estrategias de mediación citadas.

Es importante indicar que para entender la politización de los recursos literarios se debe dimensionar la literatura como producto de la construcción humana, caracterizada por su fundamentación social. Lo anterior, se relaciona con lo mencionado por Saramago (2003), quien afirma que la literatura y la transformación social son categorías que se encuentran intrínsecas en la producción textual. Sobre esta misma idea, D'Alton (2007) afirma lo siguiente:

Si la literatura es una labor transformadora, que opera en el lenguaje, lo será también en la dimensión más general de la cultura, por la íntima conexión entre esta y aquel. Es en esta dimensión general, entonces, donde debemos buscar las ganancias que nos hacían falta: ¿qué aporta la literatura como práctica cultural? [...] la literatura es la utilización plena del lenguaje, entonces la literatura necesariamente debe ser la explotación de los significados culturales en su plenitud. (pp. 79-81. Destacado original de la autora)

En esta misma línea, Cros (2009) señala que no se puede considerar a la persona escritora fuera de la sociedad, ya que la elaboración de los textos se enmarca dentro de las condiciones socioeconómicas, culturales y políticas en que se encuentra inmersa. Dicho carácter social de la literatura provoca que se encuentre permeada por un conjunto de discursos sociales, construidos desde diferentes escenarios de la sociedad, tales como las clases dominantes, las agrupaciones religiosas, los movimientos sociales, los medios de comunicación, entre otros.

Van Djik (1999) profundiza en un elemento presente en todos los textos: los discursos. Según este autor, se pueden entender como un fenómeno práctico, social y cultural que aporta elementos para comprender la utilización discursiva más allá de la percepción del lenguaje como una serie ordenadas de palabras. Si bien, la literatura puede reproducir discursos basados en los sistemas de dominación; también puede convertirse en un recurso de mediación pedagógica que promueva un cambio en la enseñanza. Este último fin orientó la estrategia didáctica del presente ensayo.

Sumado a lo anterior, el "Cadáver exquisito poético" representa solo un ejemplo de las formas en cómo se pueden politizar los géneros literarios. Cabe indicar que, en este ensayo, la politización es entendida como la injerencia de la política, es decir, la visibilización de los intereses subyacentes, en áreas que se consideraban ajenas a esta (Medrano, 2017). Por consiguiente, cuando se habla de una lectura politizada de la literatura, implica partir de una dimensión ética y política, que trascienda la concepción estética e inmanente del texto.

En este punto, resulta pertinente señalar que dentro de la lista de lecturas recomendadas por el Consejo Superior de Educación (2017), se incorporaron textos con el fin de politizar la realidad experimentada por grupos poblacionales históricamente oprimidos. Uno de dichos textos es el poema "Rotundamente negra" (1994), de la escritora costarricense Shirley Campbell Barr; en donde se intenta reivindicar la identidad de las personas negras desde su propia experiencia: 
Poema 3. Rotundamente negra

$\begin{array}{ll}\text { Me niego rotundamente } & {[\ldots] \text { Y me niego absolutamente }} \\ \text { a negar mi voz } & \text { A ser parte de los que callan, } \\ \text { mi sangre y mi piel } & \text { de los que temen, } \\ \text { y me niego rotundamente } & \text { de los que lloran. } \\ \text { a dejar de ser yo } & \text { Porque me acepto } \\ \text { a dejar de sentirme bien } & \text { rotundamente libre } \\ \text { cuando miro mi rostro en el espejo } & \text { rotundamente negra } \\ \text { con mi boca [...] } & \end{array}$

Fuente: Campbell (1994, p.143)

La incorporación de este tipo textos dentro de los programas de estudio es el resultado de los aportes de docentes y asesores que han insistido en la trascendencia de convertir la literatura en un espacio para analizar de forma crítica la realidad, y no exclusivamente como un producto estético; tal y como se intenta en esta ocasión. Antes de finalizar, se pueden señalar otros ejemplos de textos costarricenses en donde interesa fortalecer el pensamiento crítico: La mujer víctima y cómplice (1981), de Yadira Calvo, Palabras indelebles de poetas negras (2018), de Shirley Campbell y Delia McDonald, Limón Blues (2002), de Ana Cristina Rossi, Impúdicas (2016), de Arabella Salaverry, Los infiernos de la mujer y algo más (1992), de Rima de Vallbona.

Finalmente, el "Cadáver exquisito poético" pretende que el estudiantado se acerque y conozca otras realidades mediante la articulación entre la enseñanza, el aprendizaje, la literatura y el contexto. Lo anterior, representa una ruptura epistemológica con la creencia de la literatura como algo ajeno y sublime que nunca forma parte de nuestras actividades prácticas cotidianas, ya que se transforma en un elemento cercano y cimentado en sus experiencias personales. Del mismo modo, este objetivo forma parte de una consolidación y la conexión entre las subjetividades, las visiones del mundo y los compromisos colectivos que debe potenciar la educación liberadora.

\section{SINTESIS Y REFLEXIONES FINALES}

La educación debe comprenderse como producto de la interacción humana, a su vez, vinculada a un contexto social, político, cultural y económico determinado. Por consiguiente, es fundamental que el cuerpo docente mantenga una postura de apertura a procesos de reflexión que permitan discutir sobre la importancia de construir proyectos pedagógicos y estrategias didácticas que respondan a las demandas específicas del contexto. En el proceso es fundamental tomar en cuenta el papel que juega la potencialización de la creatividad en las prácticas educativas.

El "Cadáver exquisito poético" representa un primer esfuerzo para llevar a cabo los fundamentos, principalmente los metodológicos, que sustentan la pedagogía contestataria (Robles, 2020); pues se considera que uno de los principales retos que tienen las pedagogías contemporáneas se relaciona con la puesta en práctica dentro de los escenarios educativos. Por tanto, al ser una propuesta inacabada y, a su vez, adecuada a un campo de estudio específico, se espera que pueda ser replanteada y aplicada en diversos contextos, a partir de los objetivos pedagógicos y curriculares definidos por las personas que facilitan los procesos de enseñanza y aprendizaje. 
En esta misma línea, se insta al cuerpo docente a socializar las estrategias didácticas que diseña e innova en sus escenarios educativos; como se pretende en esta oportunidad. Lo anterior, se debe llevar a cabo con el fin de propiciar procesos reflexivos dentro de la práctica docente.

Además, se considera que las implicaciones pedagógicas identificadas se fortalecen cuando son sometidas al análisis colectivo, ya que se permite una realimentación. Por tanto, si bien, en el ensayo se señalan una serie de implicaciones pedagógicas y críticas, estas se encuentran abiertas a recibir los aportes que permitan la reformulación de los aspectos pertinentes.

Por último, en lo que respecta a la Enseñanza del Español, es medular que los docentes partan de la concepción de la literatura como resultado de un proceso sociocultural y, al mismo tiempo, condensadora de discursos opresores y liberadores; ya que a partir de esta idea permite el surgimiento de esfuerzos a nivel metodológico acompañados de la planificación de estrategias didácticas, en donde se politice la realidad, tanto a nivel macro como micro. Del mismo modo, esta politización brinda herramientas analíticas al estudiantado en el debate de la realidad y, a su vez, rompe con perspectivas tradicionales al encauzar la educación hacia una transformación social.

\section{REFERENCIAS}

Álamo, A. (2017). ¿Qué es un Cadáver exquisito literario? Recuperado de http://www.lecturalia.com/ blog/2017/09/19/que-es-un-cadaver-exquisito-literario/

Aldana, C. (2011). Pedagogía para nuestro tiempo. Enfoque vivencial para estudiantes. Ciudad de Guatemala: Editorial Piedra Santa.

Arqueros, G., Pérez, C. y Richard, N. (1990). Cadáver exquisito. Chile: Ojo de Buey.

Breton, A. (1924). Primer manifiesto surrealista. Barcelona: Labor.

Breton, A. (2003). Diccionario abreviado del surrealismo. Madrid: Ediciones Siruela.

Calvo, Y. (1981). La mujer víctima y cómplice. San José: Editorial Costa Rica.

Campbell, S. (1994). Rotundamente negra. San José: El Arado.

Campbell, S. y McDonald, D. (2018). Palabras indelebles de poetas negras. Heredia: EUNA

Consejo Superior de Educación. (2017). Acta Ordinaria No.36-2017. Recuperado de http://www.cse.go.cr/ file/1714/download

Cros, E. (2009). La sociocrítica. Madrid: Arco Libros.

D’Alton, C. (2007). Lengua y literatura. Ensayos didácticos. San José: EUNED.

De Vallbona, R. (1992). Los infiernos de la mujer y algo más. Madrid: Ediciones Torremozas.

Departamento Ciencias de la Educación. (2018). Programa del curso ED0012 Didáctica General. (Documento inédito). Universidad de Costa Rica. San Ramón, Alajuela.

Flecha, R. (2004). La Pedagogía de la Autonomía de Freire y la Educación Democrática de Personas Adultas. Revista Interuniversitaria de Formación del Profesorado, 18(2), 27-43. Recuperado de https:// www.redalyc.org/pdf/274/27418203.pdf

Freire, P. (1982). Pedagogía del oprimido. Buenos Aires: Siglo XXI.

Freire, P. (2006). Pedagogía de la autonomía. México: Siglo XXI.

Giroux, H. (2003). Pedagogía y política de la esperanza. Teoría, Cultura y enseñanza. Buenos Aires: Amorrortu Editores. 
González, T. (2018). La mujer en el arte: objeto de representación y sujeto creador. Logroño: Universidad de la Rioja.

Hart, S. (2018). El Cadáver exquisito de César Vallejo. Revista de Investigación del Centro de Estudios Vallejianos, 1(1), 325-348. Recuperado de https://discovery.ucl.ac.uk/id/eprint/10080010/10/Hart_El\%20 cad\%C3\%A1ver\%20exquisito\%20de\%20C\%C3\%A9sar\%20Vallejo_VoR.pdf

Jiménez, C. (1997). La lúdica como experiencia cultural. Etnografía y hermenéutica del juego. Bogotá: Editorial Magisterio.

Maza, A. et al. (2008). Construyendo. En: J. Perezchica, Antología El Cadáver exquisito: la creación colectiva como fin (pp. 36-37). México: Literatura Libre.

McLaren, P. (2012). La pedagogía crítica revolucionaria. El socialismo y los desafíos actuales. Buenos Aires: Ediciones Herramienta.

Medrano, A. (2017). La politización de la sociedad y de la vida. Recuperado de https://goo.gl/qkoqnR

Ministerio de Educación Pública (MEP). (2017). Programa de Estudio de Español Tercer Ciclo y Educación Diversificada. San José: Ministerio de Educación Pública.

Moreira, M. (2005). Aprendizaje significativo crítico. Indivisa: Boletín de estudios e investigación, 15(6), 83-101. Recuperado de http://ve.scielo.org/scielo.php?script=sci_arttext\&pid=S1010-29142009000300013

Ocampo, J. (2008). Paulo Freire y la pedagogía del oprimido. Revista Historia de la Educación Latinoamericana, 10(2), 57-72. Recuperado de https://www.redalyc.org/pdf/869/86901005.pdf

Oliva, P. (2017). Las mujeres y el arte como forma propia de deconstrucción: Un debate implícito. Revista Rupturas, 7(1), 51-74. Recuperado de https://www.scielo.sa.cr/pdf/rup/v7n1/2215-2989-rup-7-01-00051.pdf

Oreamuno, Y. (1938). ¿Qué hora es? Repertorio Americano, 18(1), 1-10.

Ortega, P. (2009). La Pedagogía Crítica: reflexiones en torno a sus prácticas y sus desafíos. Pedagogía y Saberes, 31(2), 26-34. Recuperado de https://revistas.pedagogica.edu.co/index.php/PYS/article/view/659

Picado, F. (2006). Didáctica General: Una perspectiva integradora. San José: EUNED.

Ramírez, J. y Solano, S. (2018). Los desafíos del lector. San José: Editorial Arlekín.

Robles, K. (2018). Estrategia Didáctica: Politizando los recursos literarios: En búsqueda de una poesía transformadora. (Documento inédito). Universidad de Costa Rica. San Ramón, Alajuela.

Robles, K. (2019). Pedagogía contestataria: propuesta para enfrentar las relaciones de poder en el escenario educativo. Revista Ensayos Pedagógicos, 14(2), 45-63. Recuperado de https://www.revistas.una.ac.cr/ index.php/ensayospedagogicos/article/view/13062

Rossi, A. (2002). Limón Blues. San José: Editorial Costa Rica.

Saba, R. (2019). Jugando en la catedral: Cadáver exquisito, novela encadenada y más allá. En: M. Alegre y J. Nascimbene, El imperio de Donald Dworkin: Ensayos de filosofía moral, política y jurídica (pp. 187-213). Buenos Aires: Universidad de Buenos Aires.

Salaverry, A. (2016). Impúdicas. San José: Uruk Editores.

Sánchez, A. y Valladolid, C. (2008). Dos voces. En: J. Perezchica, Antología El Cadáver exquisito: la creación colectiva como fin (pp. 47-57). México: Literatura Libre.

Saramago, J. (2003). Literatura y transformación social. Revista Psicogente, 2(11), 65-69.

Van Djik, T. (1999). El discurso como interacción en la sociedad. Barcelona: Gedisa.

Van de Velde, H. (2014). Construyendo escenarios educativos, basados en cooperación genuina. Estelí: AbacoenRed.

Zúñiga, M. (2017). La estrategia didáctica: Una combinación de técnicas didácticas para desarrollar un plan de gestión de riesgos en la clase. Revista Educación, 41(1), 1-18. Recuperado de https://revistas. ucr.ac.cr/index.php/educacion/article/view/17786 